\title{
A concept of smart multiaxial impact damper made of vacuum packed particles
}

\author{
Piotr Bartkowski ${ }^{1, *}$, Robert Zalewski ${ }^{1}$ \\ ${ }^{1}$ Institute of Machines Design Fundamentals, Warsaw University of Technology, Narbutta 84, 02-254 \\ Warsaw, Poland
}

\begin{abstract}
The conceptual design of smart crash energy absorber made of Vacuum Packed Particles (VPP) is presented in this work. This structure is composed of granular material inside a plastomer sleeve. The absorber can change the flexural stiffness and as a result the amount of dissipated energy depending on the impact conditions. The special properties of the granular structure create the possibility of various applications as different working modes can be used. When the pressure inside is equal or higher than atmospheric pressure the system behaves similarly to a liquid, otherwise it has properties similar to viscoplastic solid. The physical properties of the structure may be rapidly changed by adjusting the pressure inside the system. Due to these properties Vacuum Packed Particles is considered to be a smart material.
\end{abstract}

Keywords: Adaptive Structure, Crashworthiness, Smart Materials

\section{Introduction}

The topic of controllable materials and structures, so-called "intelligent materials" or "smart structures," has been thoroughly studied over the last years, focusing on mechanical or electrical characteristics which are easy to change during the operation of the system. However, the greatest progress has been made over the last two decades. Materials such as electrorheological (ER) and magnetorheological (MR) fluids, piezoelectrics, electrostrictive ceramics or sensors based on fiber optics are relatively well understood and widely commercialized $[1,2,3]$. On the other hand, materials such as shape memory polymers, magnetostrictive materials, conductive polymers, or micro-machined electromechanical systems are not well understood yet and are in early stages of commercialization.

This paper deals with a new type of smart structures, clearly belonging to the second group, which are systems which mechanical characteristics such as shape, stiffness, or damping, are easy to change and control. It is a contribution to the rapidly growing field of smart materials and it offers a simple and easy way to modify or upgrade many mechanical or structural elements for better control of their mechanical properties in a relatively economical and environmentally friendly manner.

The term Vacuum Packed Particles (VPP) refers to a structure that is composed of a plastomer granular material with granules having a diameter of about $1-5 \mathrm{~mm}$. They are

* Corresponding author: bartkowski piotr@onet.pl

Reviewers: Mariana Pajtášová, Jan Krmela 
typically semi-finished products used in the production of packaging of liquid beverages or plastic cutlery. The loose granules are enclosed in a hermetic, flexible sleeve and when air is pumped out of it, by a suitable valve mounted on the body surface, an underpressure is generated causing controllable changes in the mechanical and rheological properties of the system. A VPP typically behaves as a plastic material at the atmospheric pressure and as a solid when the underpressure is greater. They feature typical properties of a solid, such as tensile or compressive and shear strengths, damping capacity, internal "intergranular" friction and the ability to absorb energy under impact. These are all non-linear functions of the internal underpressure. It is of importance to note that all the physical and mechanical or rheological changes induced by the underpressure are fully reversible upon restoring atmospheric pressure. Indeed, such a material can almost instantaneously, only depending on the rate of the vacuum pump, be solidified and plasticized by supplying and removing air. The viscosity in the plastic state can be increased by a factor of ten or more by applying an appropriate underpressure.

Vacuum Packed Particles has been proven to be of use in the real applications, which was confirmed by several works. The most popular application build of VPP is the vacuum mattress (vacmat), which is used for immobilization of patients. Scientist proposed the VPP for building the medical pillow for stabilization of some parts of the human body [4]. The Authors of work [5] described the concept of endoscope made of VPP, which is able to change the stiffness. Besides the medical applications, Vacuum Packed Particles are applied in robotic industry, for example paper [6] showed the possibility of using VPP in manipulators and robotic gripper. Despite many useful applications, until now VPP were not used for crashworthiness applications.

The aim of this work is to present a concept of a new adaptive crash energy absorber made of the Vacuum Packed Particles. As it is possible to change the properties of this structure in the real time it is possible to classify the Vacuum Packed Particles as a smart material. The main purpose of this device is to predict the velocity of the striking object and adjust appropriate characteristic to minimalize the value of load transferred to the object. Some papers have been published on the adaptive control of structure crash characteristic $[7,8,9]$, but what distinguishes the structure proposed in this article is the material and the way of controlling it. The absorber is made of granular material closed in plastomer sleeve, which was in detail described in $[10,11]$. This structure allows to regulate the mechanical properties of the material by changing the vacuum value inside the sleeve.

\section{Smart structures for energy absorption}

The possibility to control and change the mechanical properties according to environment conditions is desirable in crash zones in cars and other vehicles. The characteristic of the crash zone in modern cars can be treated with some approximation as a linear strengthening. Due to this during crash with low speed contact forces are determined by crash boxes and as a result the acceleration has a smaller value than in a crash with high velocity. In the real situation the length of the crash zone is limited and imposed by the design space, therefore it would seem that a characteristic without strengthening but with the equal force throughout the whole length would be more appropriate. In this case the amount of the crash energy dissipated would be higher with the same peak acceleration. Unfortunately, the big disadvantage of such a characteristic is the fact that even in a crash with low speed the injuries of the passengers would be significant. Therefore it seems that the most favorable would be to regulate the force-displacement characteristic according to crash conditions. This is a solution presented in this paper, which focuses on the conceptual design of crash energy absorber which is able to change the stiffness characteristic and as a 
result the amount of dissipated energy in the real time. The mechanical properties of the device can be changed by regulating the underpressure inside the plastomer sleeve. The assumed mechanical characteristics is presented in Fig. 1.

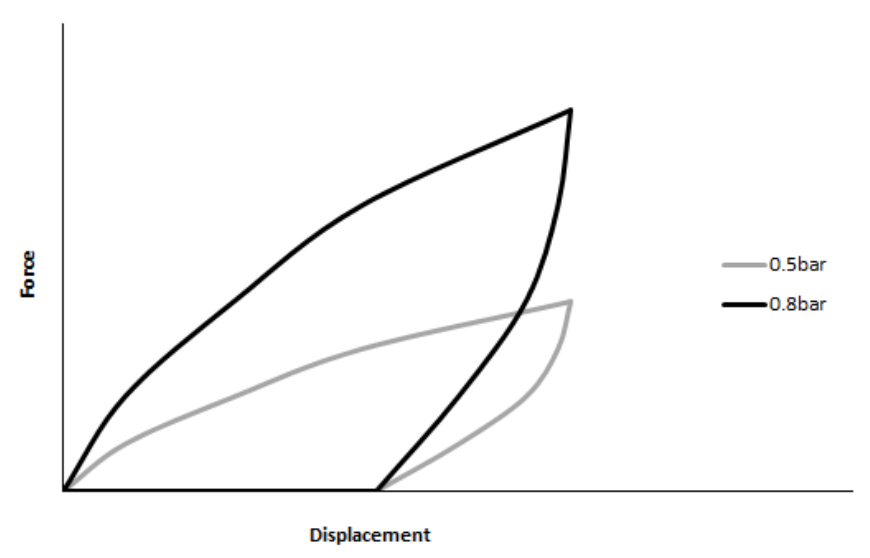

Fig. 1. Characteristics of force in funcion of displacement for the proposed absorber

The chart in Fig. 1 shows the force/displacement characteristics for two different initial values of underpressure inside the plastomer envelope. By changing the pressure parameters it is possible to regulate the amount of dissipated energy, which is equal to the area under the curve force/displacement. A damper having such a characteristics would incorporate both advantages, high crash energy absorption capability in case of the crash with high velocity and relatively low deacceleration transferred to the protected structure when the velocity is low.

\section{The concept of the absorber}

In this part of the paper the detail description of absorber design is presented (Fig. 2). The absorber consists of two aluminum plates $(2,3)$ connected by the tube (1) that is attached to plate (3) pivotally $(5,5 \mathrm{a}, 5 \mathrm{~b})$. The space between the plates is filled with granular material PS (Polistyren) (6) surrounded by rubber sleeve (4). Joint transfers the shear forces between the plates, and the moments are transmitted through the granular material. With the ability to change the interaction forces between the granules by changing the vacuum, it is possible adjust the flexural stiffness of the absorber, controlling the response of the system to impact forces. After the impact, the granulate deforms in a pseudo-plastic way. After the partial vacuum elimination, the forces between the granular material are relatively low, so the elasticity of the rubber sleeve enables the damper to return to equilibrium. It is also possible to apply additional spring element to increase the stiffness of the system. 
(a)

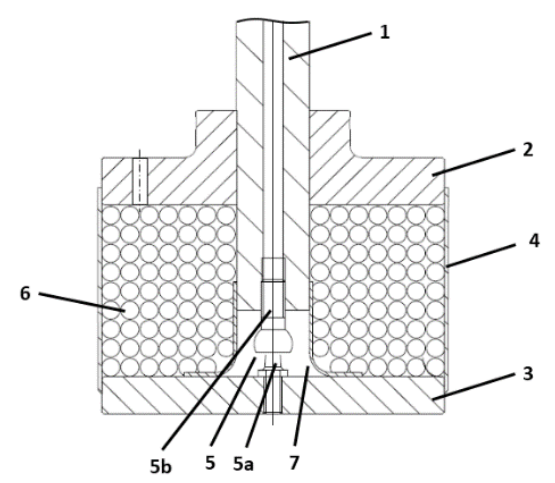

(b)

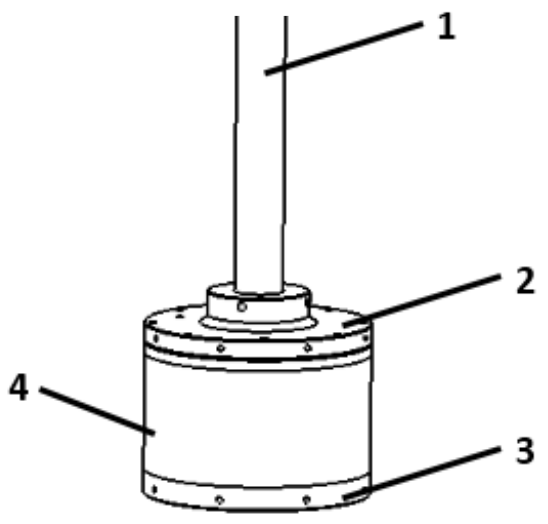

Fig. 2. The absorber design (a) cross section and (b) general view

Based on the conceptual design the prototype of the absorber was built (Fig. 3). In the preliminary configuration of the structure the plate were made with aluminum alloy and the PVC sleeve was used. This object was created to perform the preliminary experimental test allowing for verification of the concept. In this paper only the mechanical part of the device is described but the real object is additionally equipped with sensors, valves and vacuum pump with retention tank. When the velocity sensor registers the impact velocity, it changes the structure properties, by pulling out the air from the sleeve, to minimize the loads transmitted to the object while ensuring proper crash energy absorption capability.

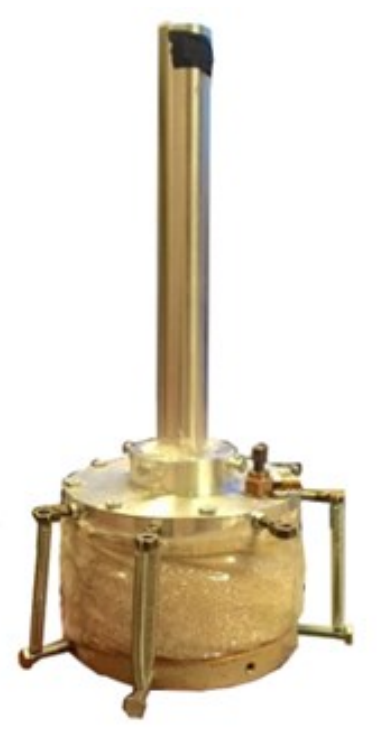

Fig. 3. The absorber prototype 


\section{Preliminary experimental research}

In order to verify the concept of the absorber the experimental research on the prototype was performed in the Institute of Machines Design Fundamentals of Warsaw University of Technology. As a preliminary experimental work, a with quasi static tests were performed on a dedicated test stand (Fig. 4). This stand consists of base (4), drive (3), displacement sensor (1) and force sensor (2), which allows to measure the force displacement characteristic of the tested object. During the experiments the vacuum inside the container was changed and measured by the pressure sensor (6). All the data were registered by a measuring card.

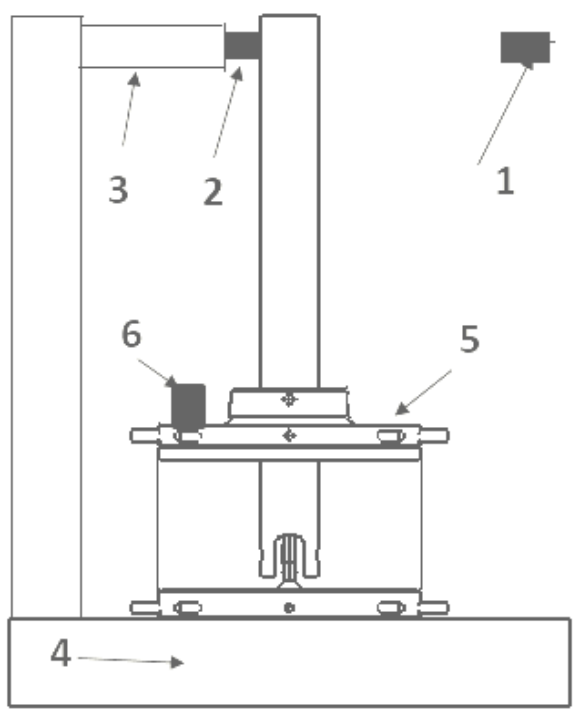

Fig. 4. Dedicated test stand

Experiments were performed for 7 underpressure values starting with $0.2 \mathrm{M} \mathrm{Pa}$ and ending with $0.8 \mathrm{MPa}$. A test for each value was repeated three times and the result were similar what is illustrated in Fig. 5.

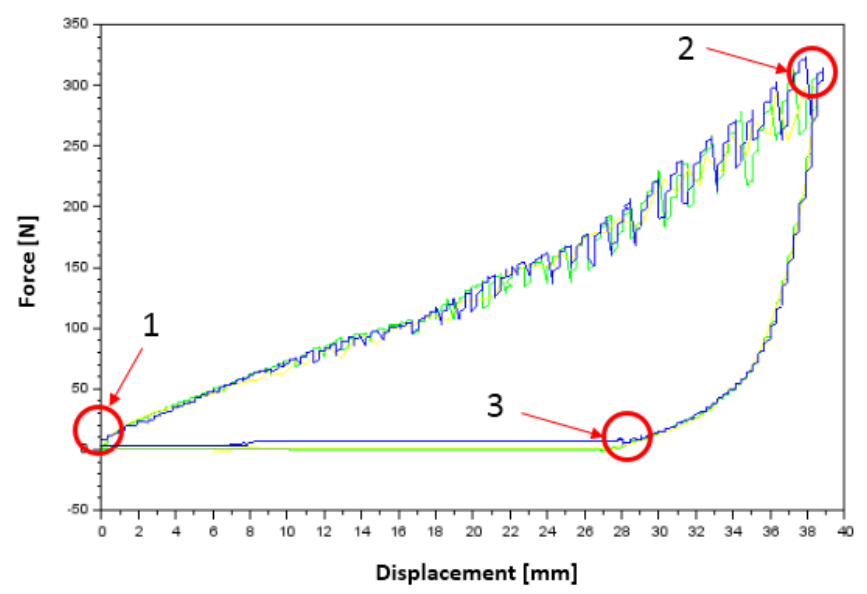

Fig. 5. The absorber characteristics 
The curve 0-1 illustrated in Fig. 6 describes the loading process of the absorber. The curve 1-2 illustrates the behavior of the absorber during unloading. In point 2 , the absorber is no longer subjected to any external force. In this case, the granulate was deformed in a plastic way, the absorber does not return to the equilibrium position. After reducing the vacuum to 0 (atmospheric pressure) the interaction between the granules are relatively small, therefore, by choosing the proper spring elements which transmit moments in parallel with the granular material, the absorber is able to return to the equilibrium state. As a result, it is possible to reuse the absorber, which is very important from the utility point of view.

The experiments were repeated for different underpressure conditions in order to check the possibility of changing the mechanical properties. The characteristics prove that it is possible to regulate the absorber properties by changing the value of vacuum inside the container. In Fig. 6 and Fig. 7 the characteristic for selected underpressure values are presented.

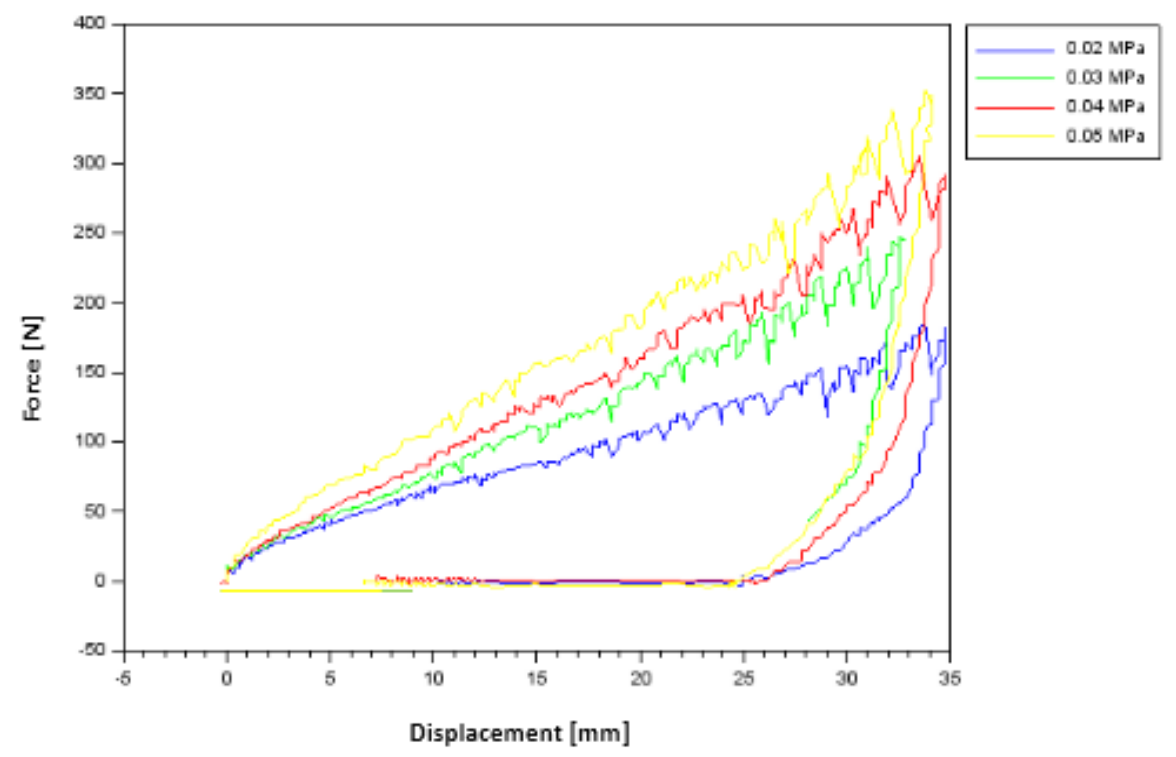

Fig. 6. Characteristic for the pressure $0.02-0.05 \mathrm{MPa}$ 


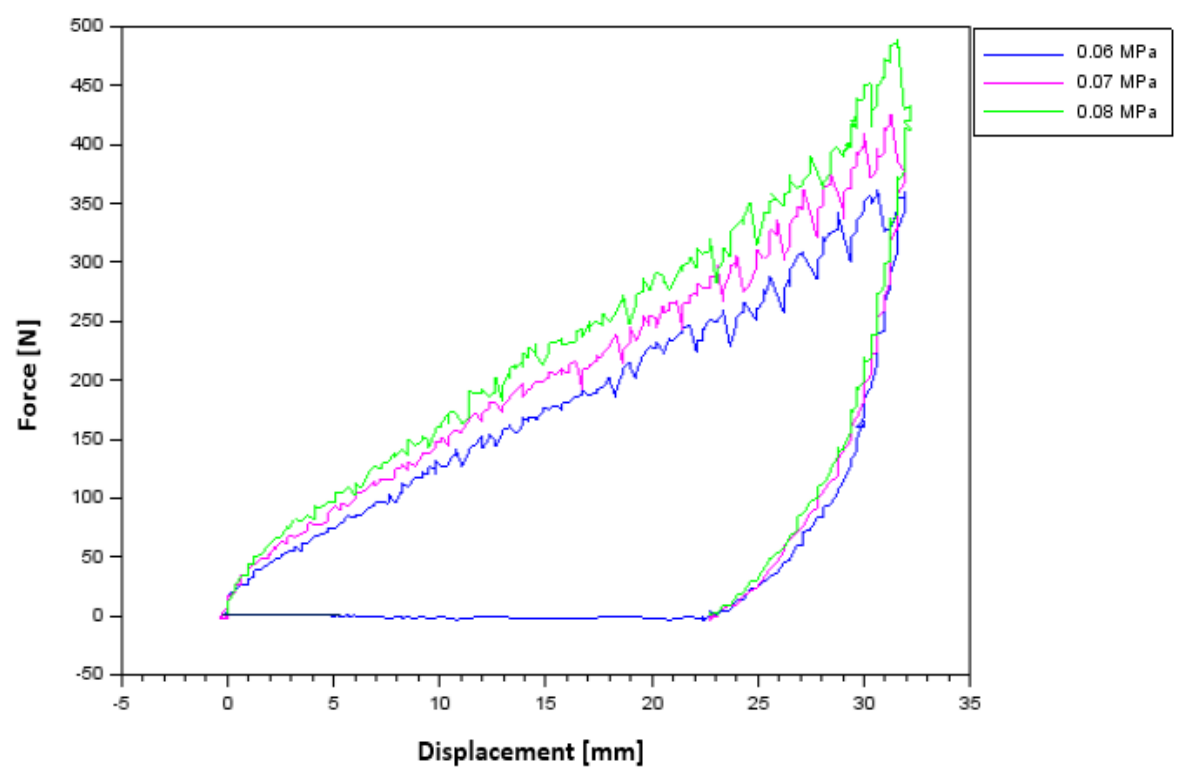

Fig. 7. Characteristic for pressure $0.06-0.08 \mathrm{MPa}$

In Fig. 8 it can be seen that the force oscillates around some value. At first, the force smoothly increases and when some value is achieved it suddenly decreases, which is probably caused by the particles relocation.

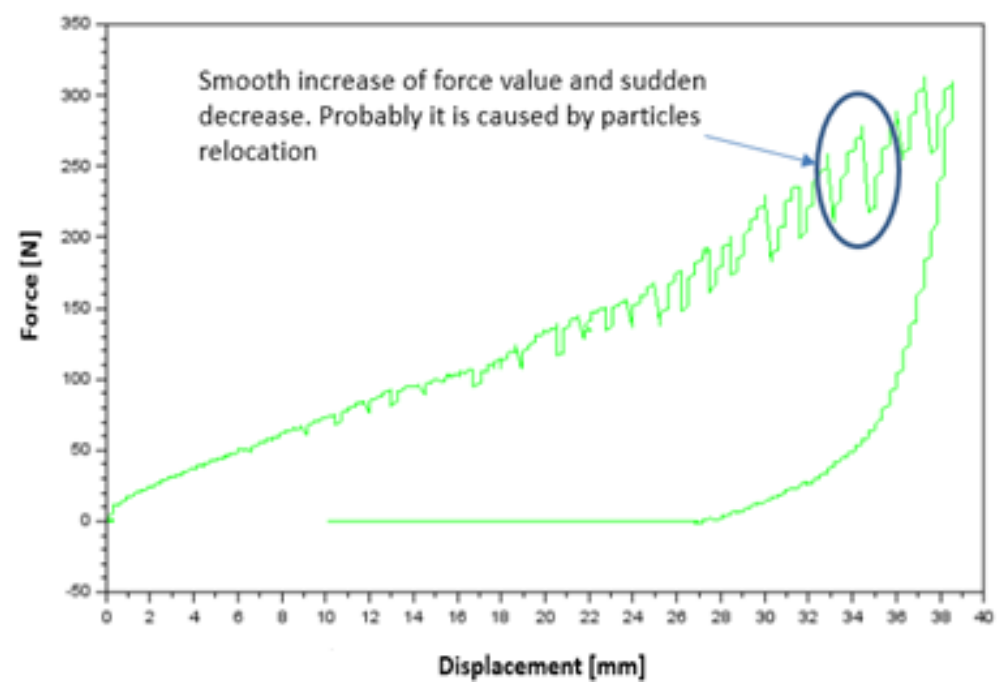

Fig. 8. The effect of the particles relocation

\section{Conclusions}

In this work the new concept of the impact energy absorber made of Vacuum Packed Particles was presented. The authors showed the basic assumption of the device and present the prototype built for performing the experiments. In the final stage of the work the preliminary results of quasi static test were presented. The experiments showed that all 
design assumption were met. It is possible to control and regulate the stiffness characteristic of the device and as a result the amount of the absorbed energy. Taking into consideration this special ability, the Vacuum Packed Particles absorber will be able to be implemented in various uses. The research showed that the device has a potential for commercialization and is worth further investigating. Vacuum Packed Particles behave similar to viscoplastic material so strain rate has the influence on their behavior. As the quasi-static test is not suitable to check all the properties of the absorber, the author is planning to perform the dynamic tests in the future. Additionally, the patent application of this object was filed in Polish Patent Office.

This work was carried out within the project no. 2016/23/N/ST8/02056 founded by National Science Centre, Poland.

\section{References}

1. P. Bartkowski, R. Zalewski, Designing process of the drone's passive safety system. New Advances in Information Systems and Technologies, Advances in Intelligent Systems and Computing 445, Springer Int. Publishing Switzerland, 729-737 (2016)

2. E. Brown, N. Rodenberg, J. Amend, et al., Universal robotic gripper based on the jamming of granular material. Proceedings of The National Academy of Sciences of the United States of America 107, 18809-18814 (2010)

3. S.R. Gorodkin, Kordonskii, Protasevich, Estimate as to the possibility of utilizing magnetorheological suspensions in compression equipment. Magnetohydrodynamics, 24 (4), 511-514 (1989)

4. C. Graczykowski, Mathematical models and numerical methods for the simulation of adaptive inflatable structures for impact absorption. Comput Struct (2015)

5. J. Holnicki-Szulc, L. Knap, Adaptive Crashworthiness Concept. International Journal of Impact Engineering (2004)

6. A.J. Loeve, O.S. Van de Ven, J.G. Vogel, P. Breedveld, J. Dankelmann, Vacuum packed particles as flexible endoscope guides with controllable rigidity. Granul Matter. 12 (6), 543-54 (2010)

7. M.D. Luscombe, J.L. Williams, Comparison of a long spinal board and vacuum mattress for spinal immobilization. Emergency Medicine Journal 20 (1), 476-478 (2003)

8. M. Ostrowski, J. Holnicki-Szulc, and P. Griskevicius, Adaptive Crashworthiness of Front-End Structure of Motor Vehicles. SAE Technical Paper (2007)

9. P. Skalski, R. Zalewski, Viscoplastic properties of an MR fluid in a damper, J. Theor. Appl. Mech. 52 (4), 1061-1070 (2014)

10. P. Wang, Z. Wang, Determination of the flow stress of a magnetorheological fluid under three-dimensional stress states by using a combination of extrusion test and FEM simulation. Journal of Magnetism and Magnetic Materials 419, 255-266 (2016)

11. R. Zalewski, T. Szmidt, Application of Special Granular Structures for semi-active damping of lateral beam vibrations. Engineering Structures (2014)

12. R. Zalewski, M. Shillor, P. Chodkiewicz, Vibrations of a mass-spring system using a granular-material damper. Applied Mathematical Modelling 40, 8033-8047 (2016) 